\title{
PERFORMANCE STUDY OF AN INTEGRATED SATELLITE/TERRESTRIAL MOBILE COMMUNICATION SYSTEM
}

\author{
MIHAEL MOHORCIC AND GORAZD KANDUS \\ Jozef Stefan Institute, Jamova 39, lo01 Ljubljana, Slovenia \\ AND \\ ENRICO DEL RE AND GIOVANNI GIAMBENE \\ Department of Electrical Engineering, University of Florence, Via S. Marta 3, 50139 Firenze, Italy
}

\begin{abstract}
SUMMARY
Terrestrial cellular networks and mobile satellite systems are expected to converge towards a future integrated satellite/terrestrial mobile communication network. Besides a system globalization, the integration of terrestrial and satellite mobile systems will lead to the unloading of the fixed part of the mobile network. This paper proposes an integrated satellite/terrestrial mobile communication system and evaluates its performance in terms of the blocking probability for new call attempts, the call dropping probability and the probability of unsuccessful call. This communication system was simulated and its performence compared with that of a stand-alone terrestrial mobile system. In the terrestrial part of the system we have considered fixed channel allocation (FCA) and dynamic channel allocation (DCA) techniques. Satellite channels can have equal or lower priority compared to terrestrial channels. The improvement of the system performance by means of satellite-to-terrestrial handovers was also estimated.
\end{abstract}

KEY WORDS: integrated mobile communication system; layered cellular structure; dynamic channel allocation

\section{INTRODUCTION}

The increasing demand of network capacity is the biggest challenge for network operators today. Because the number of radio-channels allocated to the mobile system is strictly limited, the cellular concept $^{l}$ was adopted to improve the efficiency of mobile networks. In such an approach the same channels can be reused in cells that are sufficiently separated (reuse distance), so that the co-channel interference is below tolerable levels. An additional increase in network capacity can be achieved by a further division of the territory into smaller cells, so that the channels are reused more frequently. The drawbacks of using very small cells are as follows: the location of base stations; difficulties in connectivity assurance due to fast moving mobile users and a greater number of handovers per served call.

Problems due to coverage gaps and the need to perform handovers very frequently can be overcome by the deployment of a layered cellular structure, ${ }^{2}$ in which different-sized overlapping cells are used to cover areas with different traffic densities. Therefore, future mobile communication systems will be characterized by the coexistence of several cellular layers (Figure 1); nano- and pico-cells in buildings, micro-cells in urban areas, macro-cells in suburban and rural areas, and satellite cells for the global coverage of the earth.

The quantity of traffic managed by a mobile communication system strongly depends on the channel allocation technique used, ${ }^{3,4}$ because the application of an efficient channel allocation technique can ensure a high resource reusability and increases the capacity of mobile communication systems.

Referring to the set of channels available for allocation in a cell, we may distinguish between fixed and dynamic channel allocation techniques. The fixed channel allocation (FCA) technique can only allocate channels from a set that is assigned to the specific cell. This technique blocks the calls in the overloaded cells even if there are free channels in adjacent cells. With dynamic channel allocation (DCA), channels are allocated to call demands from a common pool of resources. The only constraint for the allocation of a particular channel in a cell is the fulfilment of the minimum reuse distance among co-channel cells.

The blocking for handover requests (probability $P_{\text {hh }}$ ) is much more undesirable than the blocking of new call attempts (probability $P_{\text {ba }}$ ), because it causes the dropping of a call in progress. The probability that a served call experiences a forced termination after a certain number of successful handovers, $P_{\text {drop, }}$, is another significant parameter to evaluate the quality of service. Taking into account the current trend of providing a wide range of users with attractive services, it is generally agreed, that the values for $P_{\text {ba }}$ and $P_{\text {drop }}$ should be in the order of few percent 


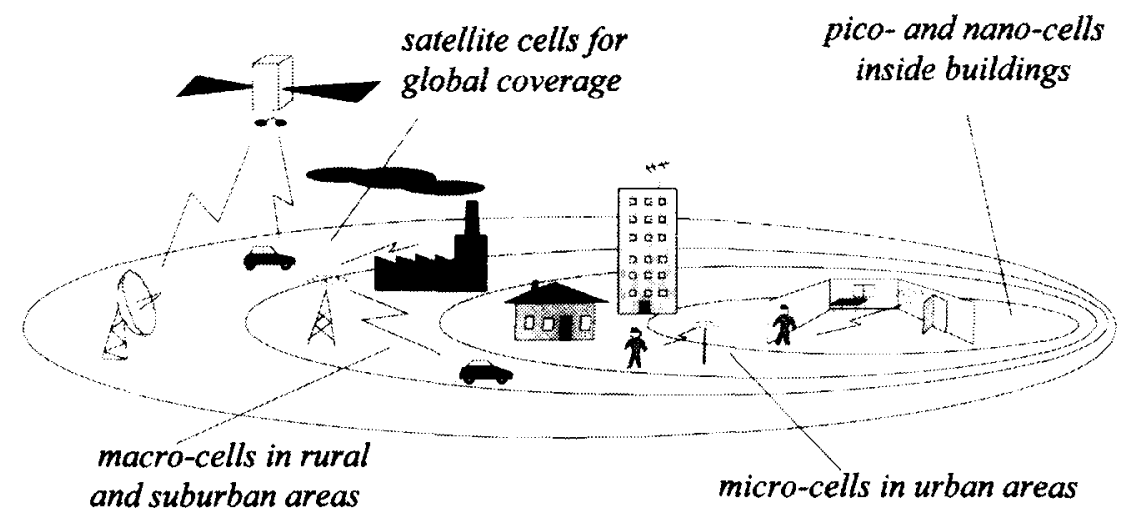

Figure 1. Layered cellular structure

and $5 \times 10^{-4}$, respectively. ${ }^{5}$ The performance comparison among different channel allocation techniques may also be carried out in terms of the probability of unsuccessful call, i.e., the probability that a call is initially blocked or it is dropped in a subsequent handover, $P_{\text {ns }}$.

Satellite mobile communication systems can be based on different satellite constellations. ${ }^{6}$ According to the altitude and the shape of the satellite orbit we may have geostationary orbit (GEO), intermediate circular orbit (ICO), highly elliptical orbit (HEO) and low earth orbit (LEO). These orbital solutions require a different number of satellites to cover all the earth or a given region.

Also, the satellite mobile communication system is based on the cellular concept, to ensure an efficient use of the assigned frequency spectrum. Each satellite covers a part of the earth's surface, and the coverage area of each satellite is further divided into satellite cells that are the footprints on the earth of antenna multi-spot-beams from satellites. ${ }^{7}$ Because satellite cells have a diameter two orders of magnitude greater than terrestrial cells, the satellite system is adequate only for low traffic density applications (e.g. electronic mail, remote database access, telephony, mobile services as a back-up system for congested terrestrial networks, etc.).

The integration of satellite and terrestrial mobile systems leads to the following advantages: system globalization; unburdening of the fixed part of the network; lower blocking probabilities and higher network capacity. These improvements can be achieved at the expense of an increased system complexity. In the integrated mobile communication system most of the traffic will be managed by the terrestrial part of the network. A satellite cell will act as an "umbrella" cell that covers many cells of a terrestrial system. Therefore, the satellite part will provide communication services to users in overloaded cells, in gaps of the terrestrial coverage, and also in areas where the terrestrial network will not be deployed at all. Thus, we can conclude that the integrated satellite/terrestrial system allows an easier planning of the terrestrial system, where reconfigurations will only rarely be required.

Previous works mainly address the performance evaluation for a stand-alone mobile communication system, that is either terrestrial ${ }^{3,4,9,10-13}$ or satellite, ${ }^{7,14}$ whereas this paper proposes the performance evaluation for an integrated system, where the satellite and the terrestrial components should co-operate to ensure a better quality of service. ${ }^{2}$ The scope of this paper is actually to study the effect of different channel allocation techniques in the terrestrial part of the network together with different satellite channel selection strategies.

A simulation model was built to estimate the performance improvement of the integrated system in comparison with a stand-alone terrestrial mobile system.

In the terrestrial network we adopted fixed or dynamic channel allocation techniques with variable assumptions for traffic loads and user mobility. For the sake of simplicity, most of the results were achieved using a simple FA DCA algorithm, but also a more complex DCA algorithm, derived from Reference 7 and here called "cost-function DCA" (CF DCA), was considered.

For the satellite part of the integrated mobile system we selected a LEO satellite system. In particular, the parameters of the IRIDIUM system ${ }^{14}$ were considered for the definition of the simulation model (see Table I).

This paper is organized as follows: Section 2 deals with the operation of the integrated mobile communication system. Sections 3 and 4 are devoted to the description of fixed and dynamic channel allocation techniques. A simulation model for the

Table I. Parameters of the IRIDIUM system

Number of satellites

Number of orbital planes

Orbital altitude

Orbital satellite velocity

Number of spot-beams per satellite
66 $780 \mathrm{~km}$ $\approx 26,600 \mathrm{~km} / \mathrm{h}$ 48 
integrated mobile communication system is described in Section 5, and simulation results are given in Section 6.

\section{DESCRIPTION OF THE INTEGRATED MOBILE COMMUNICATION SYSTEM}

In order to clarify the operation of an integrated satellite/terrestrial mobile communication system, it is convenient to introduce the following considerations and terminology. ${ }^{9}$

Let us refer to a stand-alone terrestrial system. When a new call demand is originated in a terrestrial cell, it is served by one of the terrestrial channels assigned to the base station (BS) of the cell. If there is no channel available in the BS, the new call demand is blocked and cleared from the system. A served call can be completed in the origination cell (=source cell) or it can be handed over to a BS of an adjacent cell (-target cell). When the mobile crosses the boundary between the source and the target cell while the call is in progress, a handover request is sent to the new BS. The mobile terminal becomes aware of the need for a handover when the power received from the target cell exceeds the minimum acceptable level in the receiver. From that instant and until the power received from the source cell decreases below the minimum acceptable level of the receiver, the mobile is crossing the overlap area between adjacent cells. If the handover is successfully performed while the mobile is in the overlap area, the call is continued in the target cell, otherwise it experiences a forced termination (call dropping). The extension of the overlap area is a parameter influenced by the terrain configuration, the mobile environment, the antenna characteristics and the cellular network layout. It directly affects the probability that a given handover attempt fails $\left(P_{\mathrm{bh}}\right)$. In order to keep the simulation results unaffected by this parameter, we chose the worst case: the overlap area equals zero. As a consequence, if the handover cannot be performed at the instant when the mobile requests it, the call is dropped.

Now an integrated scenario is considered. As to the resource assignment strategy, satellite channels can have the same or a lower priority level with respect to terrestrial channels. ${ }^{15}$ In the case of the same priority level, the satellite network is considered as an extension of the terrestrial network, which sees the satellite part of the system as an additional pool of resources. The choice between satellite and terrestrial channels is based only on link quality parameters. This approach leads to a worse efficiency of the satellite network, because it does not take advantage of the specific features of the satellite channels: as a matter of fact, satellite channels are more costly and more limited compared to terrestrial ones, but they can serve a much wider area. These characteristics can be better utilized by assigning a lower priority level to the satellite resources that are allocated only in case of the unavailability of terrestrial channels, regardless of its cause.

Considering the peculiarities of the satellite part of the network, a connection established on the satellite channel can be re-routed back to the terrestrial part of the network as soon as $G$ terrestrial channels are available in the current cell of the mobile user (this is the so-called "satellite-to-terrestrial handover"). The use of a value of $G$ greater than 1 avoids continuous handovers between the terrestrial network and the satellite network and a heavy signalling load to be managed by the network. However, such a solution may not use the satellite peculiarities efficiently (e.g. a satellite channel is more costly than a terrestrial one). Therefore, in this paper we considered the two extreme situations, that is $G=1$ (i.e. a call is switched back to the terrestrial network as soon as a channel becomes available in the current cell) and $G=\infty$ (i.e. a call re-routed to the satellite system cannot be switched back).

\section{FIXED CHANNEL ALLOCATION (FCA) TECHNIQUE}

In the FCA technique, system resources are divided into $N$ subsets and distributed among cells according to a regular pattern (uniform traffic case). This pattern is obtained by the spatial repetition of a cluster of $N$ cells (each cell of the cluster has a different subset of resources) so as to ensure that co-channel cells lie at a minimum reuse distance $D$. Each cell can use only the channels from its subset, although there may be free channels in adjacent cells. Such an approach leads to an inefficient use of system resources. The cluster size $N$ depends on the minimum reuse distance $D$, which is determined, in turn, by the minimum acceptable $C / I$ ratio. The relationship between the reuse distance $D$ and $[C / I]_{\min }$ is given in natural values by $(1)$, where $R$ is the cell radius and $\gamma$ is the propagation exponent ( $\gamma \approx 4$, in the terrestrial environment). ${ }^{16}$

$$
\left[\frac{C}{I}\right]_{\min } \approx \frac{\left(\frac{D}{R}\right)^{\gamma}}{6}
$$

Current analogue mobile phone systems generally require $[C / I]_{\min } \approx 18 \mathrm{~dB}(N=19)$; in the GSM the required $[C / I]_{\min }$ is reduced to about $9 \mathrm{~dB}(N=9)$, whereas in future mobile communication systems the cluster size will be equal to $3\left([C / I]_{\min }=3 \mathrm{~dB}\right)$ and even to 1 with CDMA. In this paper we adopted $N=3$ (i.e. $D=3 R$ ).

Let us assume that calls originate and end in the same cell (i.e. users are fixed, no handover is required). Then, with FCA, each cell can be modelled as a loss queuing system with a Poisson call arrival process and exponentially distibuted call 
duration times. $P_{\mathrm{b}}$ denotes the call blocking probability averaged all over the network with the weights derived from the traffic loads in cells. For uniform traffic loads, $P_{b}$ with FCA can be calculated using Equation (2), the Erlang-B formula, because the traffic load is the same in each cell. Whereas, for spatially non-uniform traffic loads, ${ }^{10}$ Equation (3) gives the blocking probability $\mathrm{P}_{b}$ averaged all over the network.

$$
P_{b}=\frac{\rho^{\mathrm{M} / \mathrm{N}}}{(M / N) !}\left[\sum_{i=0}^{\mathrm{M} / \mathrm{N}} \frac{\rho^{\mathrm{i}}}{i !}\right]^{-1}
$$

In the previous Equation (2), the variable $\rho$ represents the traffic load per cell, $M$ denotes the number of different channels in the system and $M / N$ represents the number of channels permanently assigned to a cell.

$$
P_{\mathrm{b}}=\frac{1}{L} \cdot \sum_{k=1}^{H} \frac{\rho_{\mathrm{k}}^{\left(M_{\mathrm{k}}\right)+1}}{\left(M_{\mathrm{k}}\right) !}\left[\sum_{i=0}^{M_{k}} \frac{\rho_{\mathrm{k}}^{\mathrm{j}}}{i !}\right]^{-1}
$$

In Equation (3), index $k$ denotes the variables of a generic cell $k$; moreover, $\rho_{k}$ is the traffic load in cell $k, M_{k}$ is the number of channels allocated to cell $k$, sum $L=\sum_{k}^{H} \rho_{k}$ is the total traffic load in the system with $H$ terrestrial cells. In this case, the averaged traffic load per cell is $\rho=L / H$.

\section{DYNAMIC CHANNEL ALLOCATION (DCA) TECHNIQUE}

The DCA technique can allocate any channel in any cell as long as the constraint on the reuse distance $D$ among cells using the same channel is respected. Because many channels in a cell may fulfil this constraint (they form the set of available channels in the cell), an additional algorithm has to be implemented to choose among them. Simple algorithms like the first available (FA), the nearest neighbour (NN) and the mean square (MS) were studied in Reference 10; however, more efficient algorithms can perform intra-cell handovers to optimize the channel distribution in the network. The optimization can be on local or global basis. A global optimization of the channel allocation (e.g. the DCA algorithm called maximum packing, MP) $)^{4.12}$ leads to a substantial decrease in call blocking probability, but may require a lot of channel rearrangements per served call. Therefore, a viable solution is represented by techniques that perform only a local optimization such as the priority list $(\mathrm{PL})^{13}$ or the CF DCA algorithm. ${ }^{7}$ Intra-cell handovers may take place at the origin (MP) or at the termination of a call (CF DCA and PL), or both at the origin and at the termination.

In the following, two algorithms are considered: the FA DCA algorithm without intra-cell handovers and the CF DCA algorithm that allows intra-cell handovers.

\subsection{FA DCA algorithm}

In our previous work, ${ }^{10}$ we studied DCA algorithms without intra-cell handovers; namely: FA, MS and NN. We established that the difference among them is not very significant, especially from low to medium traffic loads per cell. Therefore, we chose the simplest FA algorithm for simulation purposes; channels are scanned according to the predefined order, and a new call demand or a handover request in a cell is served by the first channel that is not used in the cells within the reuse distance.

\subsection{CF DCA algorithm}

The selection policy of an available channel heavily affects the system performance in the presence of a high traffic load, because the allocated channels are usually not only a minimum reuse distance apart, as they are in the FCA technique. The CF DCA algorithm ${ }^{7}$ chooses among available channels in a cell so as to achieve a locally optimized channel distribution. A channel rearrangement may take place at the termination of a call in a cell (this is due to the physical end of the call or to a handover) so as to pack the channels as close as possible.

First, let us distribute channels among the cells according to the FCA technique. Therefore, each cell has its own optimum set of nominal channels $F_{D}(x)$. At the instant of a call arrival in cell $x$, the set $\Lambda(x)$ of available channels in $x$ is updated. Let us denote by $I(x)$ the set of cells that lie within the reuse distance $D$ from $x$. In case of $\Lambda(x) \neq 0$, the most suitable channel to serve the request in $x, i^{\prime}$, is selected by minimization of the cost function in Equation (4).

$$
C_{x}\left(i^{\prime}\right)=\min _{\mathrm{i} \in \Lambda(\mathrm{x})}\left\{C_{x}(i)\right\}
$$

To take into account that the CF DCA algorithm preferably allocates channels belonging to the set $F_{D}(x)$, the cost contribution for the channel $\mathrm{i} \in \Lambda(x)$ due to the interfering cell $k$ is given by Equation (5), and the overall cost function for each channel $\mathrm{i} \in \Lambda(x)$ is expressed by Equation (6).

$$
\begin{aligned}
C_{x}(k, i) & =v_{k}(i)+2\left(1-q_{k}(i)\right), \forall k \in I(x) \\
C_{x}(i) & =q_{x}(i)+\sum_{k \in \mathrm{I}(x)}\left\{C_{x}(k, i)\right\}, \forall i \in \Lambda(x),
\end{aligned}
$$

where

$$
\begin{aligned}
& v_{k}(i)=\left\{\begin{array}{l}
1, \text { if } i \in \Lambda(k) \\
0, \text { otherwise }
\end{array}\right. \\
& q_{k}(i)=\left\{\begin{array}{l}
0, \text { if } i \in F_{D}(k) \\
1, \text { otherwise }
\end{array}\right.
\end{aligned}
$$


At the termination of a call on the channel $j^{\wedge}$ in cell $x$, the CF DCA algorithm computes the deallocation cost $R_{x}(j)$ for each channel $j$ allocated in cell $x$. Then, the channel $j^{\prime}$ is released in cell $x$ according to the condition in Equation (9).

$$
R_{x}\left(j^{\prime}\right)=\min _{j \in \mathrm{A}(x)}\left\{R_{x}(j)\right\}
$$

where $A(x)$ is the set of channels in use in cell $x$ at the call termination instant. If channel $j^{\prime}$ differs from channel $j^{\wedge}$, the call in progress on channel $j^{\prime}$ must be handed over to channel $\dot{j}^{\wedge}$ (i.e. intra-cell handover or channel rearrangement).

$R_{x}(j)$ is obtained as follows. Expression (10) is the de-allocation cost contribution for a channel $j \in A(x)$ due to the interfering cell $k$; note that Equation (10) is complementary to Equation (5).

$$
R_{x}\left(k_{y} j\right)=b_{x}\left(k_{y} j\right)+2 q_{k}(j), \forall k \in I(x)
$$

where

$$
b_{x}(k, j)=\left\{\begin{array}{c}
0, \text { if channel } j \text { is locked in } k \\
\text { only by the allocation in } x . \\
1, \text { otherwise }
\end{array}\right.
$$

The overall de-allocation cost function, $R_{x}(j)$, for each channel $j \in A(x)$ is given in Equation (12).

$$
R_{x}(j)=1-q_{x}(j)+\sum_{k \in \mathcal{I}(x)}\left\{R_{x}(k, j)\right\}, \forall j \in A(x)
$$

The de-allocation algorithm frees the channel that becomes available in the greatest number of interfering cells.

\section{THE SIMULATION MODEL}

Whereas the blocking probability for the FCA technique can be expressed by a closed form expression, the blocking probability for the DCA technique can be only estimated by simulations. Therefore, we built a simulation model to analyse and compare different channel allocation solutions. The simulation model, as well as the real integrated system, consists of a terrestrial and a satellite part. It enables the study of different assumptions regarding the users' mobility, traffic load, priority level of the satellite resource and satellite-to-terrestrial handovers.

In the case of DCA, the following model for the terrestrial and the satellite part of the system was used, whereas in the FCA case only a stand-alone terrestrial system was assumed.

\subsection{Terrestrial part of the simulation model}

The following simulation model ${ }^{10,17}$ was used for the terrestrial segment of the system:

- Suburban area macro-cells are considered for the terrestrial network, because they represent the layer from which calls are re-routed to the satellite system;

- The simulated terrestrial cellular network consists of $36(6 \times 6)$ hexagonal cells with a total of $M^{*}$ channels to serve call demands; in the following, this group of 36 cells will be called the area (Figure 3);

- Only voice traffic is considered;

- A one-ring interference is supposed $(D=3 R)$, which determines a reuse factor $N$ equal to 3 ;

- New call arrivals are generated according to a Poisson process independent from cell to cell; cell $k$ has an average arrival rate $\lambda_{k}$;

- Call duration is exponentially distributed, with a mean value $\mu^{-1}=100 \mathrm{~s}$;

- The traffic load in cell $k$ is given by $\rho_{k}=\lambda_{k} / \mu$;

- Mobile-to-mobile calls that require two channels to be served are considered to be about $5 \%$ of all new calls;

- If no terrestrial channel is available, the request is automatically re-routed to the satellite part of the integrated system.

In case of non-uniform traffic loads, two different traffic patterns were determined as follows. We assigned random numbers to the cells according to a uniform distribution. Two different number distributions were determined. Then, these patterns were normalized so as to have an average value equal to 1 for both cases (see Figure 2). The statistics of the two basic non-uniform patterns, namely $A$ and B, are summarized in Table II. The average traffic load per cell in the simulated area was distributed to the cells of the area by using the numbers associated to the cells according to a pattern. The performance of the system in the non-uniform case was obtained by using both schemes (A and B) and varying the average traffic load per cell from 3 to 10 Erlangs.

Pattern B presents a greater standard deviation than pattern $A$; therefore, the traffic pattern $B$ is "more non-uniform" than traffic pattern A.

As to the mobility model, we built a simulation to evaluate the statistical distribution for the mobile residence time in a cell. We assumed that the starting position of the mobile unit in the source cell can be anywhere, and that it can move in any direction with randomly defined initial speed. During the time spent in a cell a mobile user can randomly change the speed and the direction of its motion. Moreover, the mobility scenario is that of suburban area cells: this is the layer of the terrestrial cellular structure from which calls are directly re-routed to the satellite network. We assumed that the average

\footnotetext{
*In case of integrated mobile system, the terrestrial part of the network uses $M=36$ channels. Whereas, in the case of a standalone terrestrial cellular system the previous value has been increased with the average number of satellite resources available per simulated area, in order to make a fair comparison (see Section 6).
} 

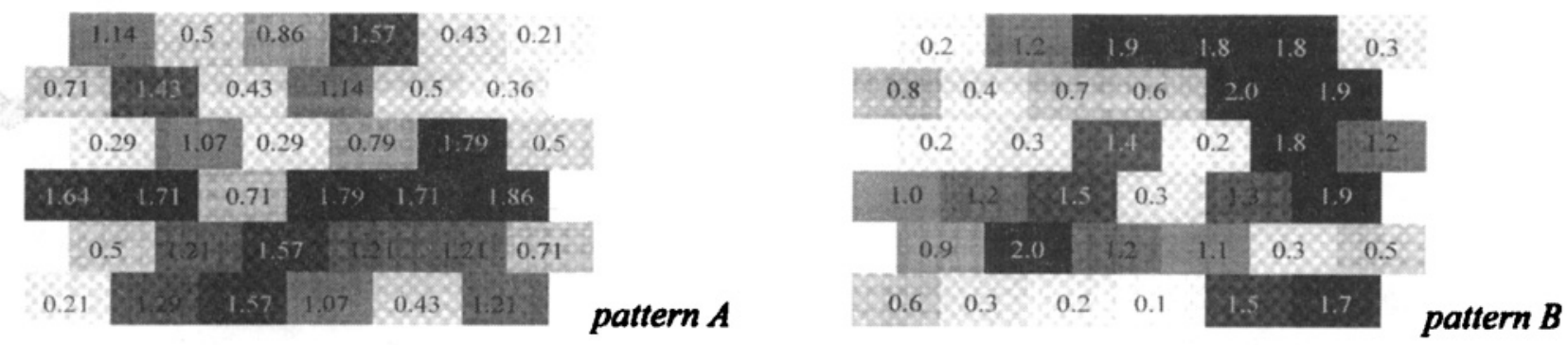

Figure 2. Non-uniform basic patterns $\mathrm{A}$ and $\mathrm{B}$

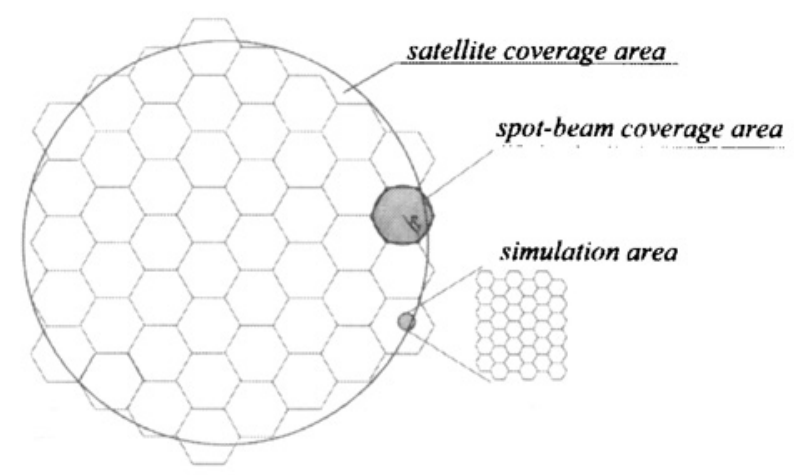

Figure 3. Cellular layout of the simulation model

Table II. Statistics of the patterns A and B

\begin{tabular}{llc}
\hline Parameter & Pattern A & Pattern B \\
\hline Mean value & 1 & 1 \\
Standard deviation & 0.529 & 0.647 \\
Maximum & 1.86 & $2 \cdot 0$ \\
Minimum & 0.210 & $0 \cdot 1$ \\
Max/min ratio & 8.857 & $20 \cdot 0$ \\
\hline
\end{tabular}

mobile speed is $45 \mathrm{~km} / \mathrm{h}$ and the cell side is $3.7 \mathrm{~km}$. According to the above simulation model, the mobile user residence time in these cells was estimated to be spread according to a Gaussian distribution with mean value and variance equal to $300 \mathrm{~s}$ and $225 \mathrm{~s}^{2}$, respectively. Note that the average mobile residence time results as the time spent by a mobile moving from the centre of a cell directly towards the cell boundary.

A handover request occurs when the time spent in the cell by an active mobile user is shorter than its call duration. A handover is successful if it is possible to find an available channel in the target cell immediately, where the entire allocation procedure is repeated.

\subsection{Satellite part of the simulation model}

The satellite part of the model is based on the parameters of the well-known LEO satellite system called IRIDIUM. In addition to the parameters introduced in Table I, we considered that all spot-beam footprints have the same radius, $R_{S}=212.5 \mathrm{~km}^{7}$ Sat- ellites constitute a regularly meshed network that circulates around the earth with a constant velocity. This entails that spot-beam footprints on the earth have a speed which is much higher than the mobile users' velocity and the earth's rotation speed.

All the following aspects must be considered to estimate the number of satellite channels that are available for a given area:

1. The channels of a satellite are shared among all the 48 spot-beams of the satellite antenna. We assumed that the number of channels available per spot-beam $(C)$ equals 330 , and that any channel can be allocated to any terrestrial cell covered by the same spot-beam (according to a dynamic channel allocation).

2. As is shown in Figure 3, each satellite cell covers many simulation areas $(=6 \times 6$ terrestrial cells) and the probability that a satellite channel is used in an area is, on average, the same for all the areas covered.

3. Satellite cells are fast moving with respect to terrestrial cells.

Then, we obtained a conservative estimate of the average number $(S / 2)$ of channels available in a simulation area as follows. Let us denote by $a$ the side of a hexagonal terrestrial cell. The area of a cell is $3 \sqrt{3 a^{2} / 2}$, whereas the circular footprint with radius $R_{\mathrm{s}}=212.5 \mathrm{~km}$ covers an area on the earth that is approximately equal to $\pi R_{s}^{2}$ (approximation: flat earth). The number $n_{\text {cell }}$ of terrestrial cells covered by a satellite spot-beam is expressed by Equation (13). 


$$
n_{\mathrm{cell}}=\left[\frac{2 \pi R_{S}^{2}}{3 \sqrt{3} a^{2}}\right]
$$

Then, the average number of satellite channels available in a simulated area is obtained as follows:

$$
\frac{S}{2}=\left[\frac{36 \cdot C}{n_{\text {cell }}}\right]
$$

If we choose suburban area small cells with $a=3.7 \mathrm{~km}$, we obtain $n_{\text {cell }}=3988$ from Equation (13), and consequently, $S / 2 \approx 3$ from Equation (14). We may note that satellite channels are distributed within a spot-beam among more than 100 simulation areas, and a lot of random aspects are involved in determining the actual number of satellite channels available in a simulation area. Therefore, according to the central limit theorem, a reasonable assumption on the statistics of the number of satellite channels available in a simulated area could be given by a discrete Gaussian distribution between 0 and $S$ with a mean value equal to $S / 2$ and a standard deviation of $S / 8$.

The parameter values used for the simulations of the satellite part of the system are summarized below:

- $S=6$;

- A call re-routed from a congested terrestrial cell to the satellite system can be

(a) managed by the satellite system for all its duration $(G=\infty)$, or

(b) switched back to the terrestrial cellular network as soon as a terrestrial channel becomes available in the cell (i.e., satellite-to-terrestrial handovers), $G=1$.

Moreover, in the simulation model we assumed that a call attempt is blocked and the request is cleared from the system if no terrestrial and satellite channels are available.

\section{SIMULATION RESULTS}

In this Section, stand-alone terrestrial and integrated mobile communication systems are compared in terms of blocking probabilities. The integrated system has higher overall capacity (terrestrial and satellite channels) than stand-alone terrestrial system, but in order to obtain a fair comparison, systems with equivalent capacity were compared. The number of satellite channels of the integrated system, available in simulation area, varies in time, but the average number of channels is known and given as $S / 2$. Therefore, the number of channels of the standalone terrestrial system was increased by $S / 2$ with respect to the terrestrial segment of the integrated system.

As to the traffic assumptions, the curves denoted by $n$ represent models with non-uniform traffic loads (i.e. with patterns $\mathrm{A}$ and $\mathrm{B}$ ). The distinction between stand-alone terrestrial and integrated mobile systems is indicated by terr and int. Considering the satellite part of the mobile system, the label sat/terr indicates the possibility of satellite-to-terrestrial handovers (i.e. $G=1$ ), otherwise the label no sat/terr signifies that no satellite-to-terrestrial handover is allowed (i.e. $G=\infty$ ). The priority level of the satellite channels with respect to the terrestrial ones is specified as $e q$ or $l o$, standing for equal or lower priority, respectively.

The performance improvement of the integrated mobile system in comparison to the stand-alone terrestrial mobile system in terms of the probability of unsuccessful call $\left(P_{\mathrm{ns}}\right)$ is shown in Figure 4 for uniform traffic loads, and in Figures 5 and 6 for non-uniform traffic load patterns $A$ and B. One can notice that the FA DCA technique shows better results than the FCA technique for low and moderate traffic loads (i.e. between 6 and 7 Erlangs per terrestrial cell), although only the simple FA DCA algorithm was used. Therefore, the utilization of a more complex DCA algorithm promises even better results, as we will show later in this Section.

Moreover, one can observe that the integrated mobile system achieved better results than the standalone terrestrial mobile system, regardless of the priority level of the satellite channels and the possibility of satellite-to-terrestrial handovers. However, the lowest $P_{\mathrm{ns}}$ was obtained by the strategy with a lower priority level of the satellite channels and with satellite-to-terrestrial handovers $(G=1)$. Anyway, the strategies without satellite-to-terrestrial handovers $(G=\infty)$ and with equal priority level of the satellite channels show only slightly worse results.

The comparison of models with different assumptions about the traffic load is shown in Figure 7 for fixed and dynamic channel allocation techniques. These results prove that the best operation (i.e. the lowest $P_{\mathrm{ns}}$ ) of the mobile communication system is achieved in the case of uniform traffic load. In the real environment the traffic load is always nonuniformly distributed. In such an environment the advantage of dynamic over fixed channel allocation due to its intrinsic adaptability is evident: the FCA technique is nearly useless above $3 \mathrm{Erl} /$ cell. Furthermore, the comparison of models with the non-uniform traffic schemes $A$ and $B$ shows that the more non-uniform the traffic load is, the worse the blocking probabilities for the FCA and for the FA DCA techniques are.

We have mentioned that the performance difference between the strategies with and without satellite-to-terrestrial handovers in terms of $P_{\mathrm{ns}}$ is slight. On the other hand, the comparison of these two techniques, with separate blocking probabilities for the terrestrial and the satellite part of the network, shows that the model with satellite-to-terrestrial handovers provides a higher quality of service. The portion of blocked terrestrial calls successfully handed over to the satellite network for different traffic assumptions is shown in Figures 8, 9 and 10. 


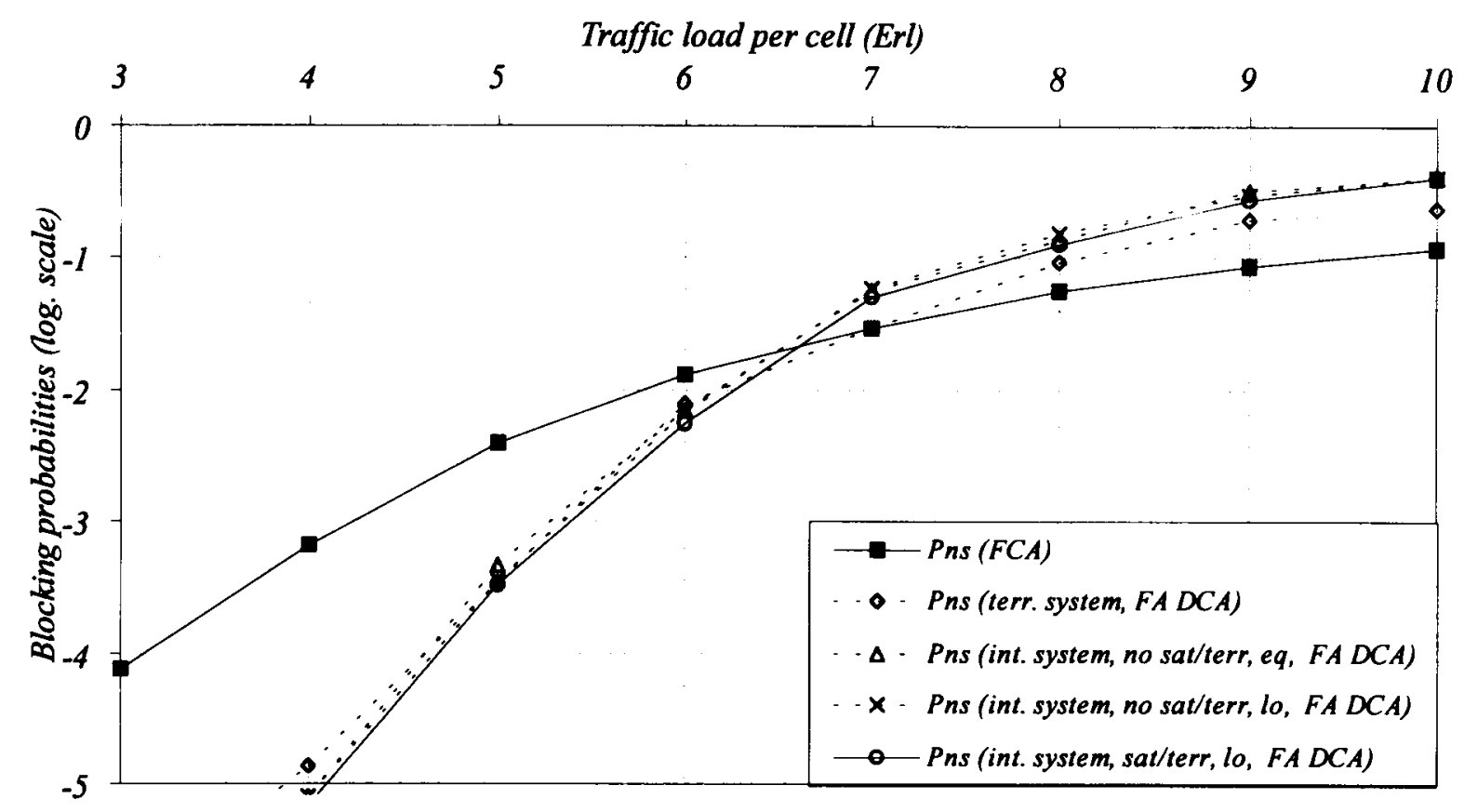

Figure 4. Blocking probabilities for uniform traffic load

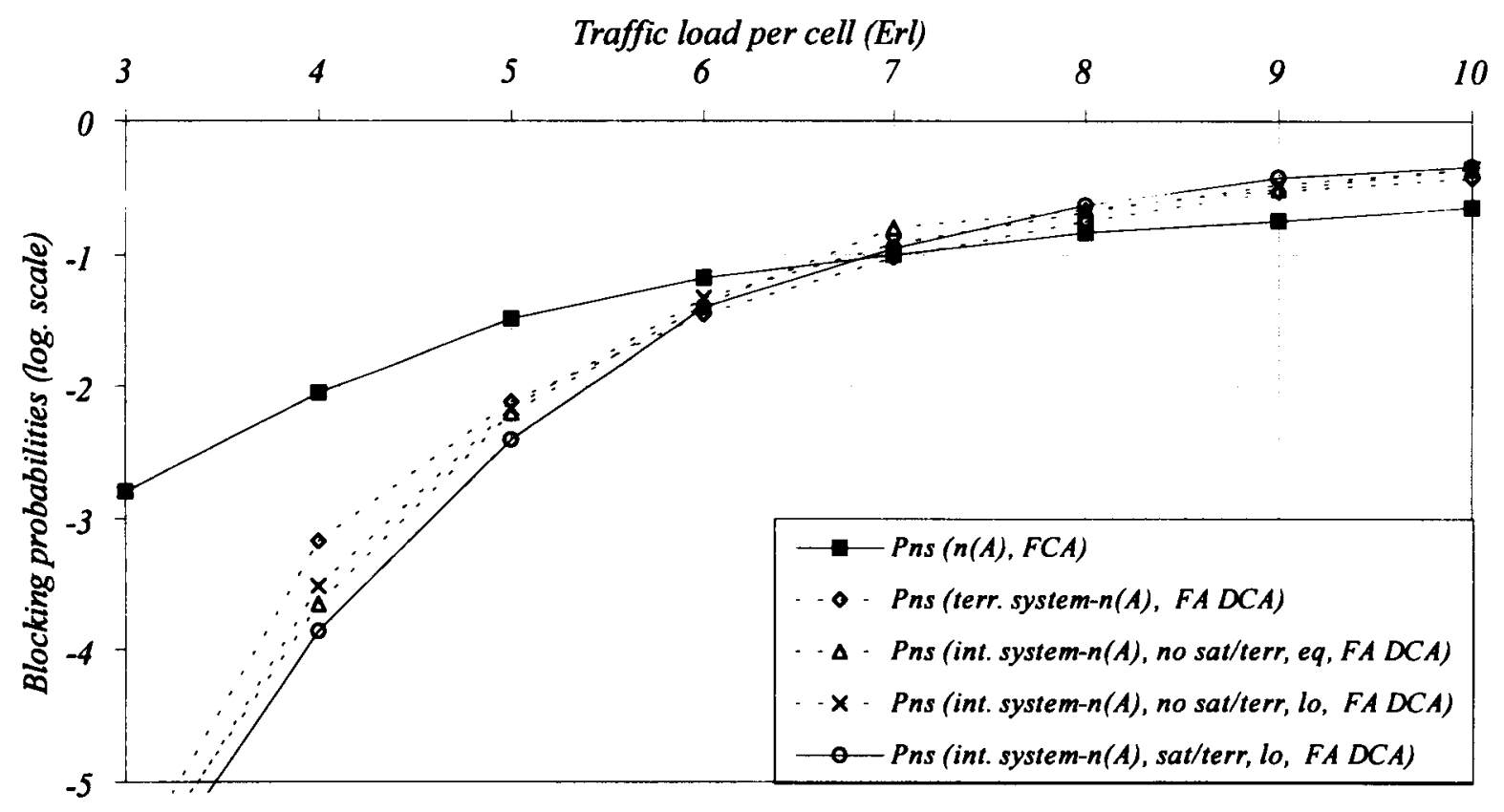

Figure 5. Blocking probabilities for non-uniform traffic load scheme A

These figures show that in the range of medium traffic loads the satellite channels are used more efficiently if satellite-to-terrestrial handovers are allowed. In the case of higher traffic loads, the difference between these models disappears, because terrestrial channels are occupied most of the time and the satellite-to-terrestrial handovers cannot take place.

Figure 11 shows the call dropping probability $\left(P_{\text {drop }}\right)$ and the blocking probability for new call attempts $\left(P_{\mathrm{ba}}\right)$ obtained by the simulation model with the following assumptions: non-uniform traffic pattern B, FA DCA in the terrestrial segment, lower priority of the satellite resources and satellite-toterrestrial handovers.

In Figures 4-11 the simple FA DCA algorithm was used. In order to attain a performance improvement, a more efficient DCA algorithm (i.e. CF DCA) was adopted to manage terrestrial resources. Therefore, the simulation results, shown in Figure 12, were obtained with the following assumptions: satellite-to-terrestrial handovers; lower priority of the satellite resources and non-uniform traffic pattern B. System performance was evaluated in terms of $P_{\mathrm{ns}}, P_{\mathrm{drop}}$ and $P_{\mathrm{ba}}$. For comparison purposes, Figure 12 also shows $P_{\text {ns }}$ for the simulation model 


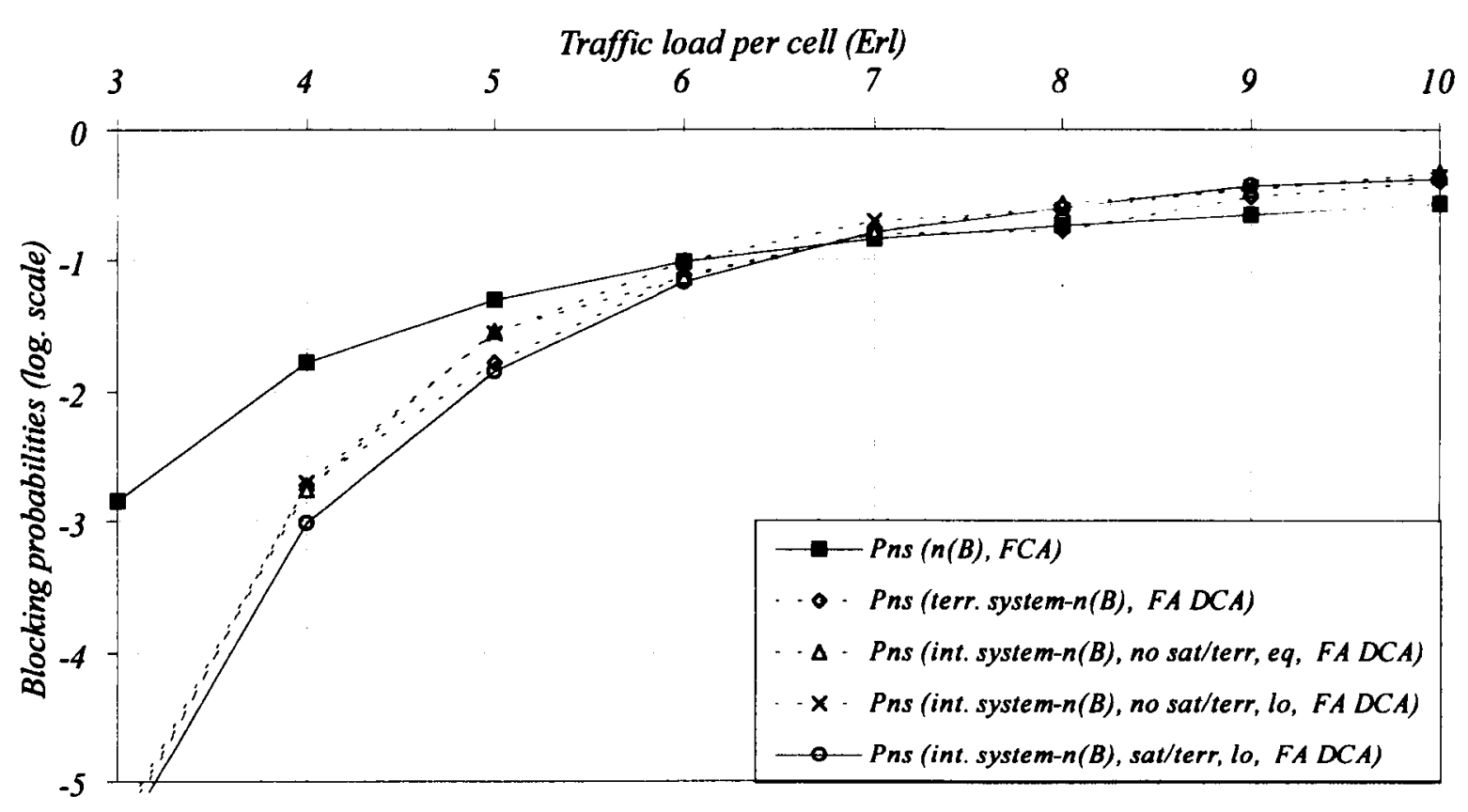

Figure 6. Blocking probabilities for non-uniform traffic load scheme B

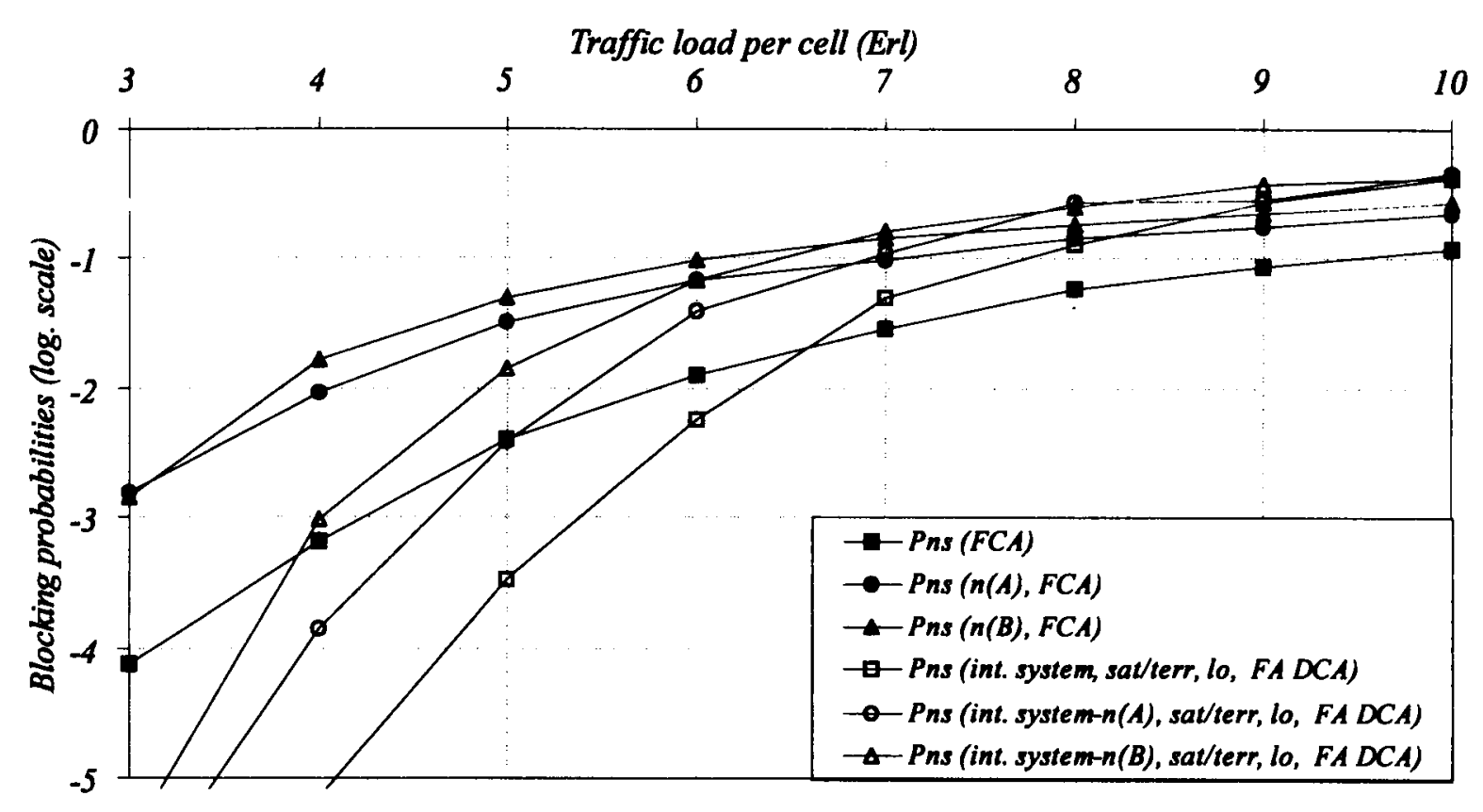

Figure 7. Comparison of models with different assumptions regarding the traffic load

with the FA DCA algorithm. Although the behaviours of $P_{\text {ns }}$ with different algorithms do not differ a lot, $P_{\text {drop }}$ is much lower for the model with the more efficient CF DCA algorithm (see Figures 11 and 12). Therefore, CF DCA can allow the fulfilment of the requirements for $P_{\text {drop }}$ and $P_{\mathrm{ba}}$ with a higher traffic load per cell than FA DCA.

Figure 13 compares results, obtained by the simulation models based on CF DCA in the presence of non-uniform traffic loads (pattern B) with and without satellite-to-terrestrial handovers, in terms of the portion of blocked terrestrial calls successfully handed over to the satellite network: CF DCA shows an improved performance with respect to FA DCA (see Figure 9).

Finally, it is important to remark that in all comparisons made (see Figures 8-10 and 13), the portion of calls refused by the terrestrial system that are handled by the satellite network increases, when satellite-to-terrestrial handovers are allowed. Therefore, this strategy permits a better utilization of satellite resources. 


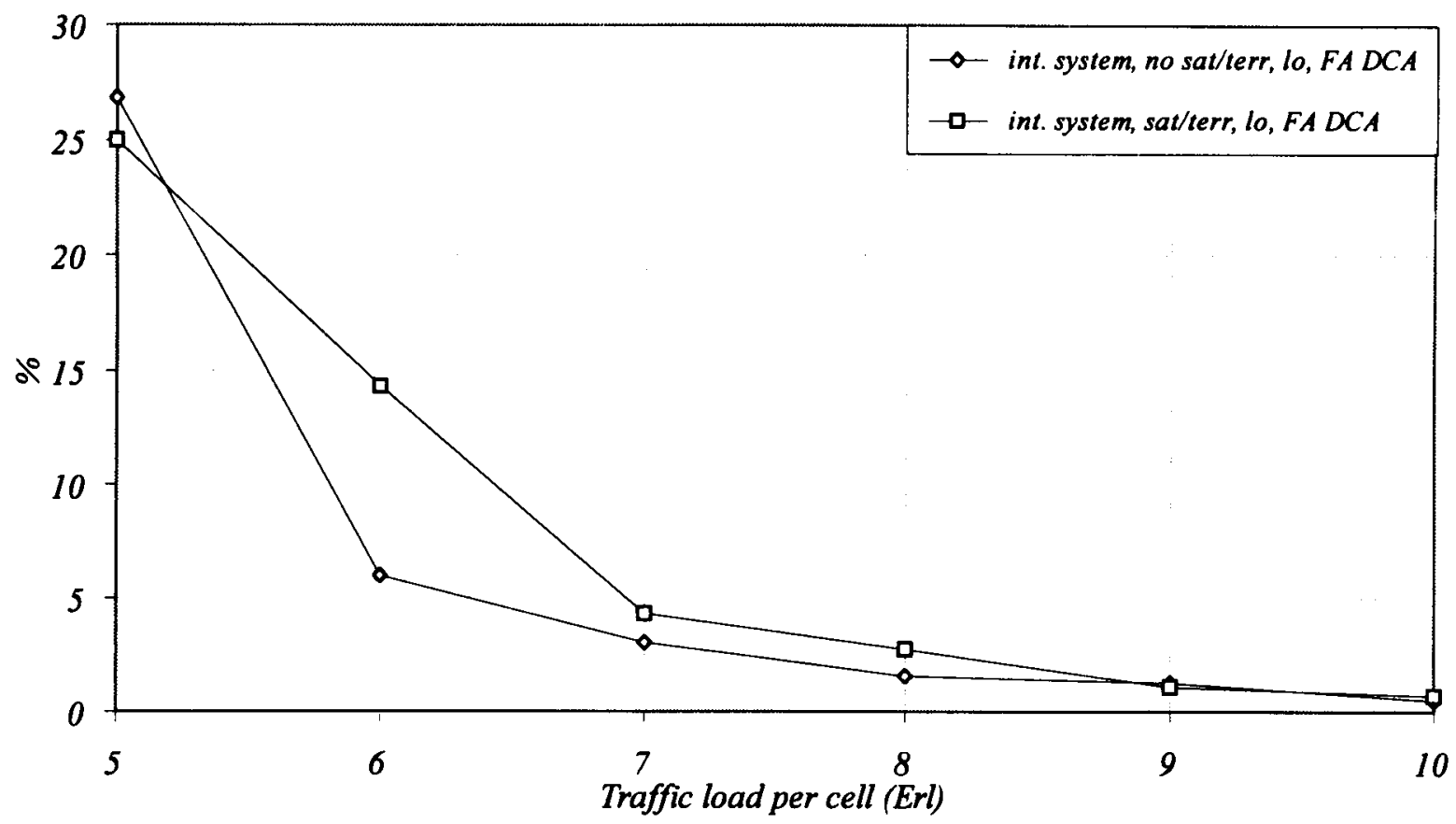

Figure 8. Portion of blocked terrestrial calls successfully handed over to satellite channels for uniform traffic load and FA DCA algorithm

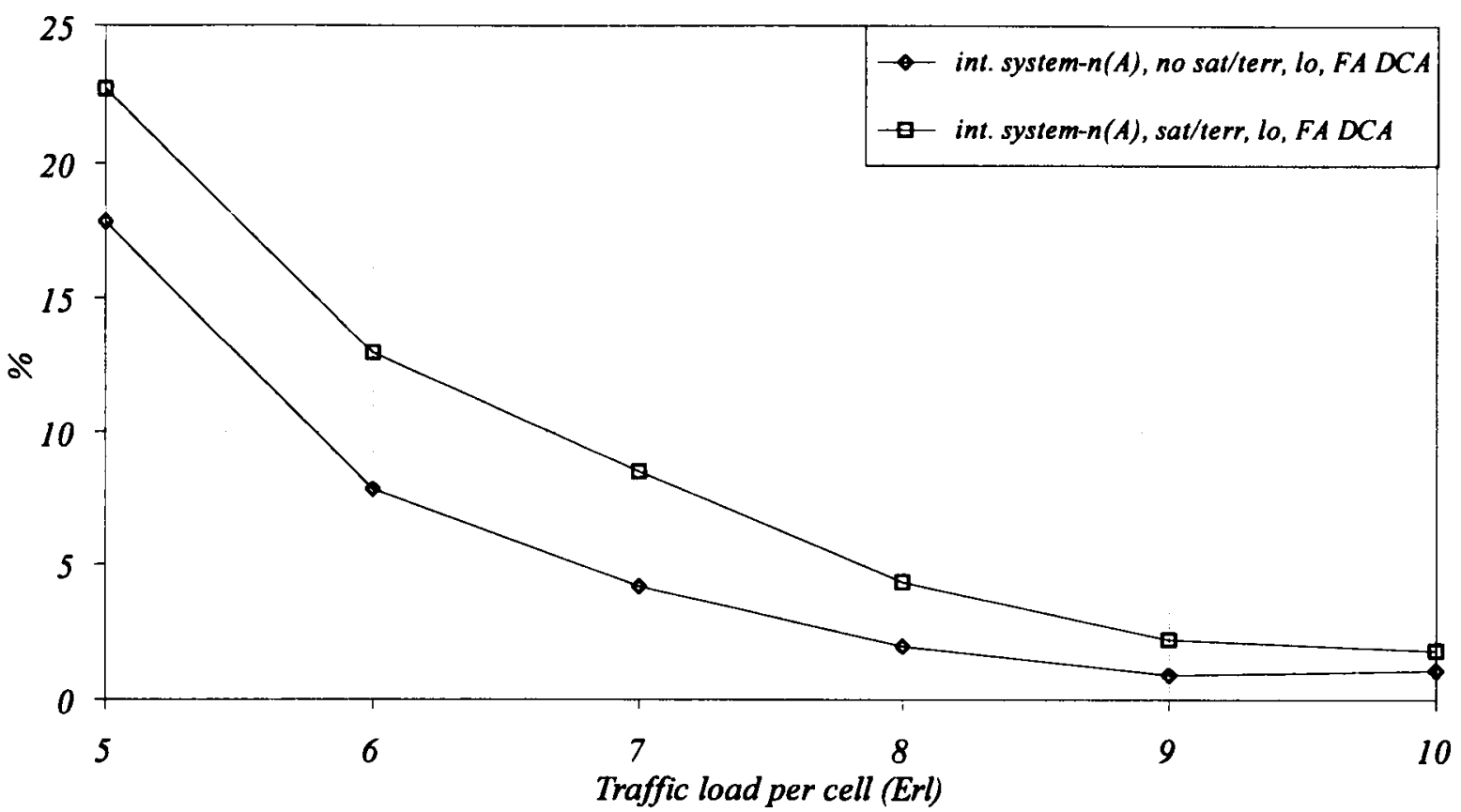

Figure 9. Portion of blocked terrestrial calls successfully handed over to satellite channels for non-uniform traffic load scheme A and FA DCA algorithm

\section{CONCLUSION}

In this paper we studied several resource management strategies for an integrated satellite/terrestrial system. These techniques were compared by simulations with those for a stand-alone terrestrial cellular system in terms of various blocking probabilities (i.e. the blocking probability for new call demands, the call dropping probability and the probability of unsuccessful call).
In the terrestrial part of the integrated network three allocation techniques were used: FCA, FA DCA and CF DCA. The last of these techniques emerged as the most promising solution, because it allows a strong reduction of the call dropping probability, one of the most important quality of service parameters for a mobile communication system from the channel allocation standpoint.

The integrated mobile communication system showed a better performance than the stand-alone 


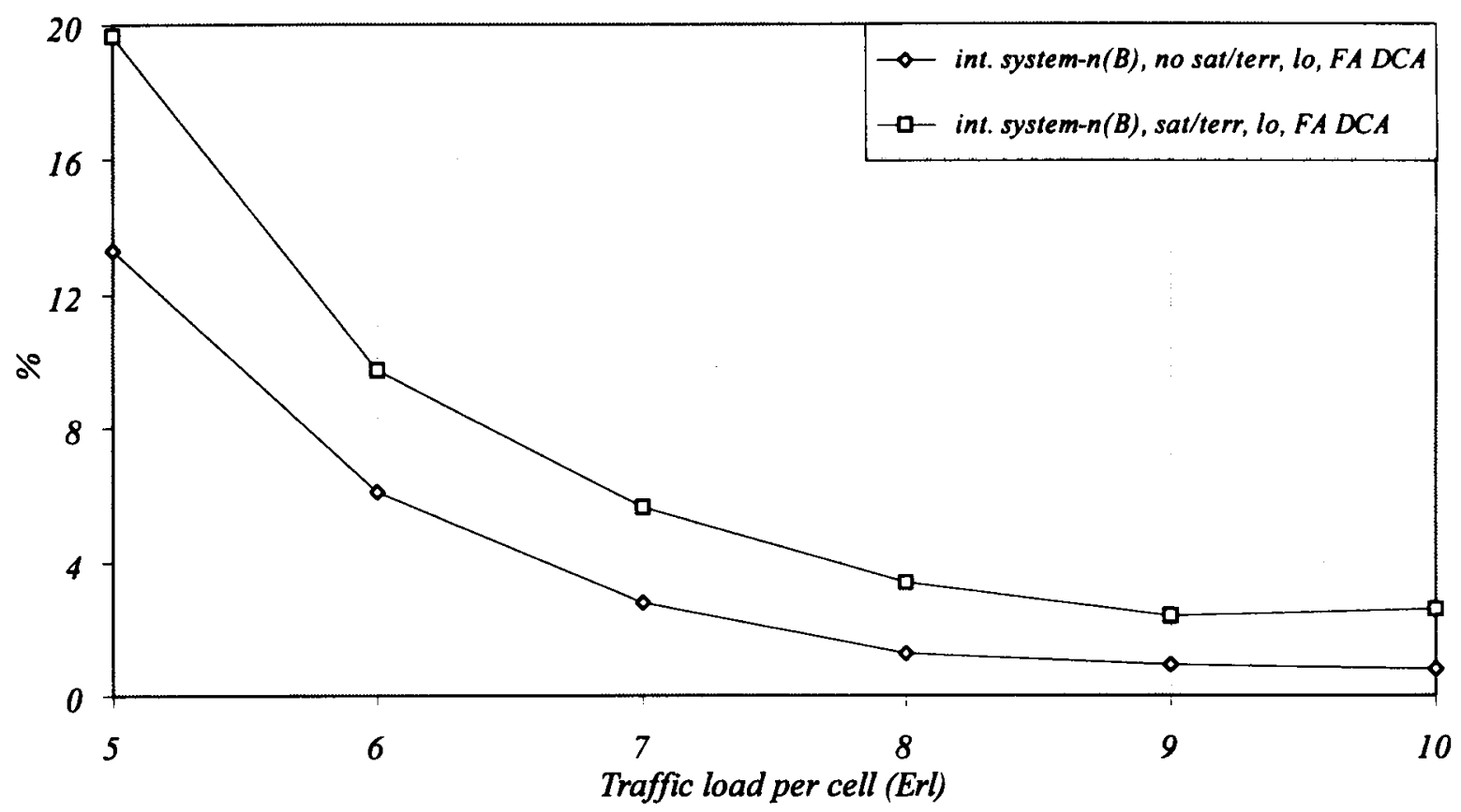

Figure 10. Portion of blocked terrestrial calls successfully handed over to satellite channels for non-uniform traffic load scheme B and FA DCA algorithm

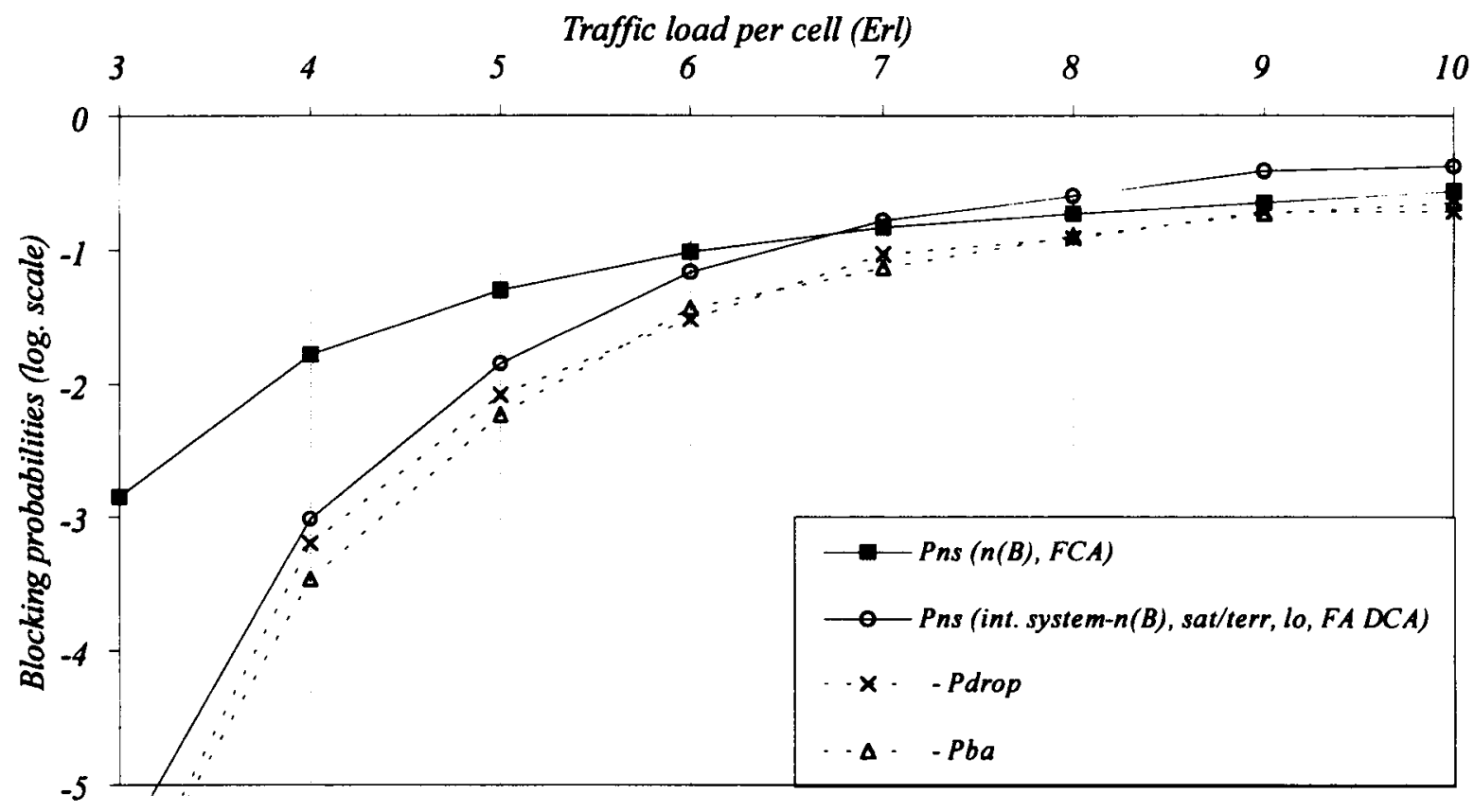

Figure 11. Probabilities of dropped calls and blocked new arrivals for FA DCA algorithm (non-uniform traffic load scheme B)

terrestrial cellular system, regardless of the allocation technique used.

We considered that a call re-routed from the congested terrestrial network to the satellite system can be switched back when $G=1$ terrestrial channels become available in the terrestrial cell of the mobile user (satellite-to-terrestrial handover). Such a technique increased the portion of call successfully handed over to the satellite network, so allowing a better utilization of satellite resources. In the real implementation one should consider the use of satellite-to-terrestrial handovers only if a certain number
$G$ of terrestrial channels were free (i.e. $G>1$ ), to avoid a continual interchange of connections between satellite and terrestrial channels.

\section{APPENDIX: LIST OF ACRONYMS AND NOTATIONS}

$\rho \quad$ Averaged traffic load per terrestrial cell

BS Base station

CF "Cost function" algorithm

D Minimum reuse distance

DCA Dynamic channel allocation 


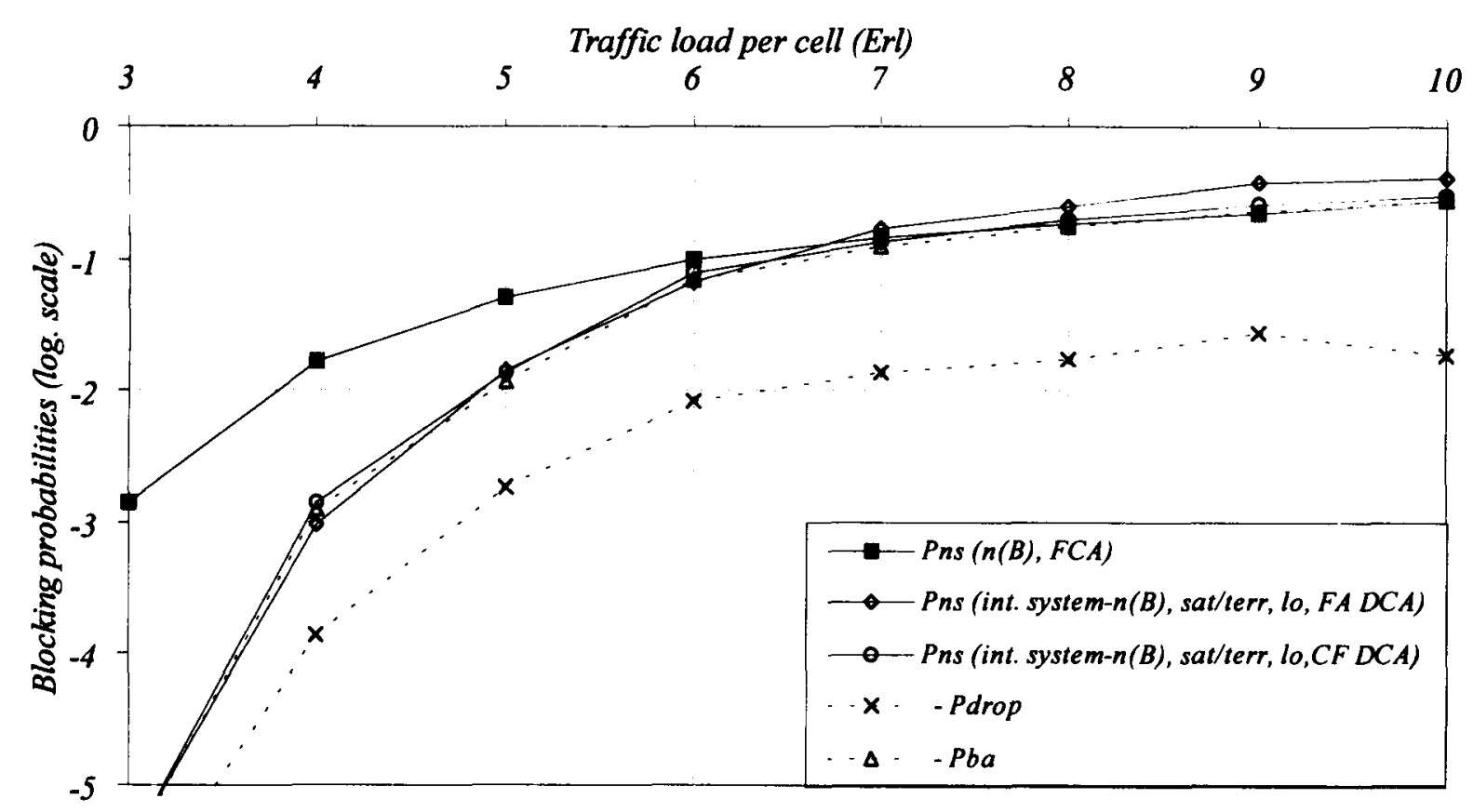

Figure 12. Probabilities of dropped calls and blocked new arrivals for CF DCA algorithm (non-uniform traffic load scheme B)

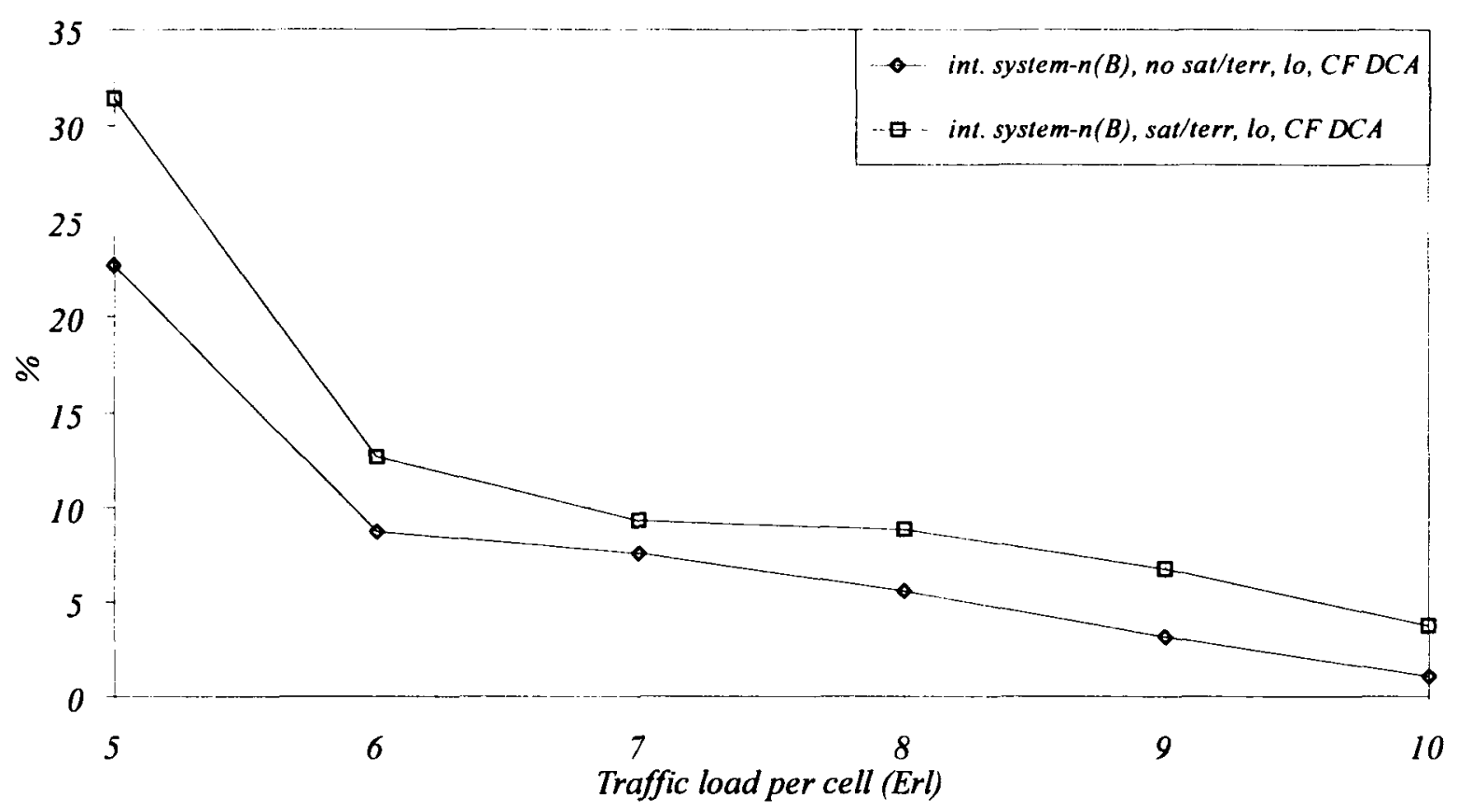

Figure 13. Portion of blocked terrestrial calls successfully handed over to satellite channels for non-uniform traffic load scheme B and CF DCA algorithm

FA "First available" algorithm

FCA Fixed channel allocation

$\mathrm{G} \quad$ Number of free terrestrial channels in a cell to allow a turnover from the satellite to the terrestrial network

GEO Geostationary orbit

HEO Highly elliptical orbit

ICO Intermediate circular orbit

LEO Low earth orbit

M Number of channels of the terrestrial system

MP "Maximum packing" algorithm
MS "Mean square" algorithm

$\mathrm{N} \quad$ Number of cells in a cluster (reuse factor)

NN "Nearest neighbour" algorithm

$\mathrm{P}_{\mathrm{ba}} \quad$ Blocking probability for new call attempts

$\mathrm{P}_{\mathrm{bh}} \quad$ Blocking probability for handovers

$\mathrm{P}_{\text {drop }} \quad$ Call dropping probability

PL "Priority list" algorithm

$P_{n s} \quad$ Probability of unsuccessful call

$R_{s} \quad$ Radius of a spot-beam footprint on the earth

$\mathrm{S}$ Maximum number of satellite channels available for a simulation area 


\section{REFERENCES}

1. V. H. MacDonald, 'The cellular concept', Bell Syst. Tech. J., 58, 15-41 (1979).

2. L. R. Hu and S. S. Rappaport, 'Personal communication systems using multiple hierarchical cellular overlays', IEEE J. Selected Areas Comm., 13, (2), 406-415 (1995).

3. D. C. Cox and D. O. Reudink, 'Dynamic channel assignment in high-capacity mobile communications systems', Bell Syst. Tech. J., 50 (6), 1833-1857 (1971).

4. P. -A. Raymond, Performance analysis of cellular networks', IEEE Trans. Commun., 39 (12), 1787-1793 (1991).

5. ITU-T Recommendations E.771, 'Network grade of service parameters and target values for circuit-switched land mobile services', ITU-T, 1994.

6. K. G. Johannsen, 'Mobile P-service satellite system comparison', Int. J. Sat. Comm., 13, (6), 453-471 (1995)

7. E. Del Re, R. Fantacci and G. Giambene, 'Efficient dynamic channel allocation techniques with handover queuing for mobile satellite networks', IEEE $J$. Selected Areas Comm., 13, (2), 397-405 (1995).

8. F. Ananasso and F. Delli Priscoli, 'The role of satellites in personal communication services', IEEE $J$. Selected Areas Comm., 13, (2), 180-196 (1995).

9. D. Hong and S. S. Rappaport. 'Traffic model and performance analysis for cellular mobile radio telephone systems with prioritized and nonprioritized handoff procedures', IEEE Trans. Veh. Techn., VT-35, (3), 77-92 (1986).

10. G. Kandus and M. Mohorcic, 'Call blocking performance for dynamic channel allocation strategies in micro-cellular mobile communication systems', in Proc. IEEE 2nd Symposium on Communications and Vehicular Technology in the Benelux, pp. 216-221, Louvain-la-Neuve, Belgium, November 2-3, 1994.

11. S. Tekinay and B. Jabbari, 'A measurement-based prioritization scheme for handovers in mobile cellular networks', IEEE J. Selected Areas Comm., 10, (8), 1343-1350 (1992).

12. D. Everitt and D. Manfield, 'Performance analysis of cellular mobile communications systems with dynamic channel assignment', IEEE J. Selected Areas Comm., 7, (8), 1172 1180 (1989)

13. A. Baiocchi, F. Delli Priscoli, F. Grilli and F. Sestini, 'The geometric dynamic channel allocation as a practical strategy in mobile networks with bursty user mobility', IEEE Trans. Veh. Techn., 44, (1), 14-23 (1995).

14. M. Werner, A. Jahn, E. Lutz and A. Böttcher, 'Analysis of system parameters for LEO/ICO-satellite communication networks', IEEE J. Selected Areas Comm., 13, (2), 371networks',
381 (1995).

15. E. Del Re, 'A coordinated European effort for the definition of a satellite integrated environment for future mobile communications', IEEE Communication Magazine, 34, (2). 98 104 (1996).

16. W. C. Y. Lee, 'Spectrum efficiency in cellular', IEEE Trans. Veh. Techn., 38, (2), 69-75 (1989).

17. M. Mohorcic and G. Kandus, 'Modelling of micro-cellular mobile communication systems', in Proc. 13th IASTED International Conference-Applied Informatics, pp. 193-195, Igls, Austria, February 21-23, 1995.

Authors' biographies

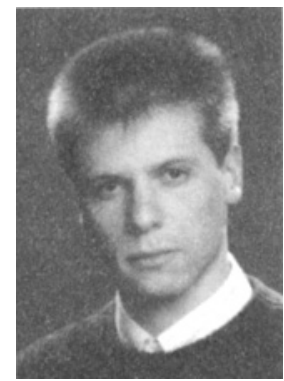

Mihael Mohorcic received his B.S. degree in electrical engineering from the University of Ljubljana, Ljubljana, Slovenia, in 1994. After his basic education he joined the Jozef Stefan Institute as a research assistant in the Telecommunications Department. He is currently a second year M.Sc. student of electrotechnics at the University of Ljubljana. His research interests include mobile communication systems with a concentration in channel allocation techniques, performance evaluation and integration of mobile satellite networks with terrestrial networks.

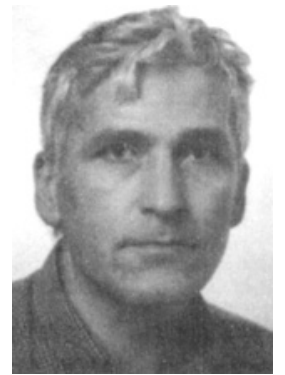

Gorazd Kandus received his B.S., M.S., and Ph.D. degrees in electrical engineering from the University of Ljubljana, Ljubljana, Slovenia, in 1971, 1974 and 1991, respectively. After working with the Institute of Transportation and Faculty of Mechanical Engineering he joined the Jozef Stefan Institute in 1979. His current research interest includes design and simulation of mobile telecommunication systems. He is the head of the Telecommunications Department at the Jozef Stefan Institute and Associate Professor at the Faculty of Electrical Engineering. In 1978 he received the Fulbright Grant and spent one year at Worcester Polytechnic Institute, Worcester, MA. as a postgraduate researcher. In 1993 and 1995 he was a visiting scientist at the University of Karlsruhe, Germany. Dr Kandus is a member of the Institute of IEEE and Upsilon Pi Epsilon Honor Society in the Computing Sciences.

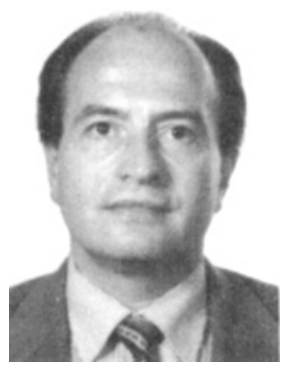

Enrico Del Re was born in Florence, Italy, in 1947. He received the Dr Ing. degree in electronics engineering from the University of Pisa, Pisa, Italy, in 1971. Until 1975 he was engaged in public administration and private firms, involved in the analysis and design of telecommunication and air traffic control equipment and space systems. Since 1975 he has been with the Department of Electronics Engineering of the University of Florence, Florence, Italy, first as a Research Assistant, then as an Associate Professor, and since 1986 as Professor. During the academic year 1987-1988 he was on leave from the University of Florence for a 9 month period of research at the European Space Research and Technology Centre of the European Space Agency, The Netherlands. His main research interests are digital signal processing, digital transmission techniques, and communication networks, on which he has published more than 150 papers, in international journals and conferences. He is the Co-editor of the book "Satellite Integrated Communications Networks" (North-Holland, 1988), one of the authors of the book "Data Compression and Error Control Techniques with Applications" (Academic, 1985) and the editor of the book "Mobile and Personal Communications" (Elsevier, 1995). He received the $1988 / 89$ premium from the IEE (U.K.) for the paper "Multicarrier demodulator for digital satellite communication systems". He is the head of the Digital Signal Processing and Telematics Laboratory of the Department of Electronic Engineering of the University of Florence. Prof. Del Re is a Senior Member of the IEEE, a member of the AEI, the European Association for Signal Processing (EURASIP) and has been included in the edition of Who's Who in the World.

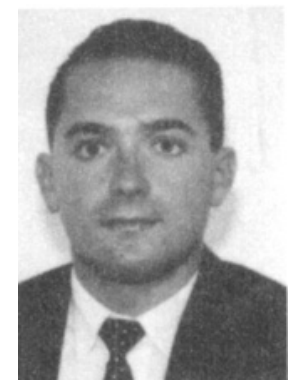

Giovanni Giambene (S'94) received the $\mathrm{Dr}$ Ing. degree in electronics from the University of Florence, Florence, Italy, in 1993. Since 1994, he has been with the Electronic Engineering Department, University of Florence, as a Ph.D. student in telecommunications and informatics. He also supplied the task of Technical External Secretary of the European Community Project COST 227

"Integrated Space/Terrestrial Mobile Networks". His research interests include: mobile cellular communication networks, personal communication services, channel allocation techniques and queuing theory. 